\title{
Automation of Tank Level Using Plc and Establishment of Hmi by Scada
}

\author{
Rishabh Das ${ }^{1}$, Sayantan Dutta ${ }^{2}$, Anusree Sarkar ${ }^{3}$, Kaushik Samanta ${ }^{4}$ \\ ${ }^{I}$ Department of Electrical Engineering UIT, BU, India \\ ${ }^{2-4}$ Department of Applied Electronics and Instrumentation Engineering UIT, BU, India
}

\begin{abstract}
Theproposed system provides an analysis of the simulation and components required for the implementation of an automated level control system by the help of Programmable Logic Controller (PLC).Supervisory Control And Data Acquisition(SCADA) was established and the HMI was created. The proposed model can effectively supervise level control in multiple tanks. Three level sensors were used to provide the level data to the PLC. PLC used this data to take the required decisions and thereby turning ON and OFF a pump. A manual switch was also provided to override the automatic system. The SIMATIC S7-300 universal controller was used as the main decision making module. The system was implemented in SCADA to create the required Human Machine Interface (HMI). Modifications can be made by using float sensors model which would effectively provide the correct level but cost would increase and vibration of the sensor might disrupt the result, our model effectively counters those shortcomings.
\end{abstract}

Keywords: Automation, SCADA, PLC,HMI, Tank-level Control.

\section{Introduction}

A Programmable Logic Controller, PLC or Programmable Controller is a digital computer used for automation of electromechanical processes [1]-[2]. Unlike general-purpose computers, the PLC is designed for multiple inputs and output arrangements, extended temperature ranges, immunity to electrical noise, and resistance to vibration and impact. Programs to control machine operation are typically stored in battery-backedup or non-volatile memory. In our project we used SIEMENS S7- 300 PLC [3].S.Ghosh et, al has effectively designeda bottle filling plant using PLC [4] and A.Saha et, al gives a proposed system that efficiently controls a cement factory using PLC[5]. A Centralized PLC Automation Control in Painting Line of Steel Plant is shown byHao, L. and Ruilin[6].Our Proposed system can be divided into three main modules- sensing, decision making and implementation. The PLC also communicates the status of the entire system through a Human Machine Interface or virtual reality supervision. Four sensors are used to implement the system. These sensors detect the presence of water. The readings of the sensors are used by the PLC to take the required decision.

Finally the decision is implemented by the PLC through a relay switch. The PLC communicates the present status of the system through the IM port to the computer which is accessible by the HMI. The ladder logic was implemented in SIMANTIC manager and the HMI was created by WINCC Explorer.

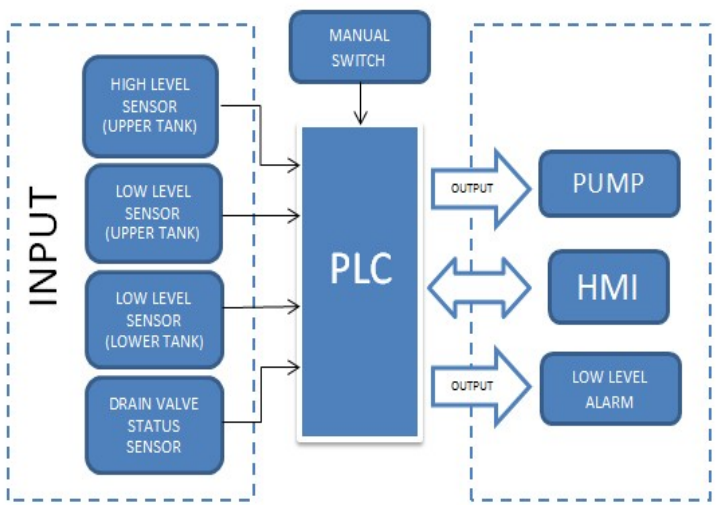

Fig.1 Block Diagram of the System

The proposed system will control and monitor the liquid level of the tank continuously and will ensure that a sufficient level of water is maintained. This system can be used ubiquitously in industrial application. It can be used to prevent industrial accident by overfilling of any open container, to prevent overfilling of any closed container thereby creating overpressure condition. The high number of the input output port of the PLC 
will enable this single system to control large number of tanks single handedly. Leakage can also be monitored. Block diagram of the system is shown in Fig 1.

The system can be divided into four main parts:-

\section{System Component Description}

2.1 Level Sensor

2.2 PLC(Programmable Logic Controller)

2.3 Relay and Motor

2.4 HMI(Human Machine Interface)

These are the four main modules of the system. The level sensor communicates the present level of the tank to the PLC. The PLC decides whether to turn the motor ON or OFF. The status of the system is communicated to the computer by MPI and is viewed and remotely controlled by the user through the HMI.

\subsection{Level Sensor}

Inductive proximity sensors were used to detect the presence of water in the tank. These sensors detect magnetic loss due to eddy currents that are generated on a conductive surface by an external magnetic field. An AC magnetic field is generated on the detection coil, and changes in the impedance due to eddy currents generated on an object are detected. When the object enters this electromagnetic field which appears at the active face of the switch, the field gets reduced and the switch turns ON or OFF. The main advantage of this sensor is that it can sense the object without touching it, so this sensor can be fixed outside the water. The internal circuit diagram of the switch is shown in Fig.2.

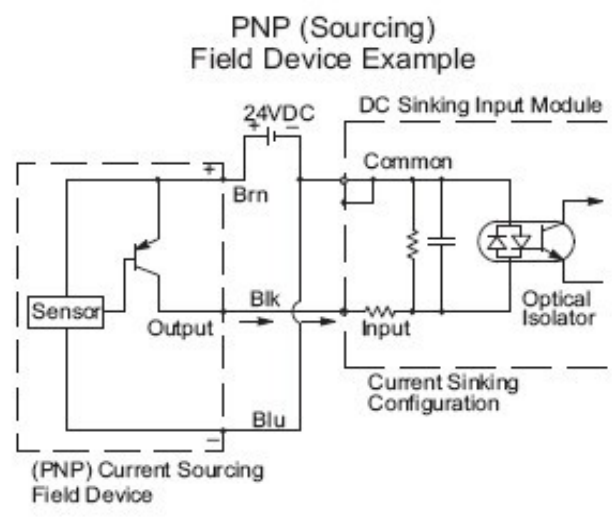

Fig. 2 Proximity Sensor internal diagram

\subsection{PLC (Programmable Logic Controller)}

This serves as the main control unit of the system. The ladder logic is prewritten on a non- volatile memory. The ladder logic was implemented in Siemens SIMANTIC manager. On basis of this logic the PLC takes its decisions. In our project Siemens S7-300 was used. The S7-300 enables space-saving and modular configurations.In a single-tiered configuration, S7-300 can support $256 \mathrm{I} / \mathrm{O}$, and in multi-tiered configurations up to $1024 \mathrm{I} / \mathrm{O}$. In distributed configurations with PROFIBUS DP,65536 I/O connections are possible (up to 125 stations, such as ET 200M via IM 153) [7]. The slots are freely addressable, that is, there are no slot rules. So a single S7-300 if deployed in an industry can automate and monitor a large number of tanks. The PLC was interfaced by to the computer via IM

\subsection{Relay and Motor}

A relay is an electrically operated switch. The relay considered in our system uses an electromagnet to operate a switching mechanism mechanically. Any compatible relay can be used with our system. The motor on the other hand was used to pump the water from the underground tank to the overhead tank. The relay converts the DC output of the PLC into a signal compatible to effectively control the motor being used.

\subsection{HMI (Human Machine Interface)}

The Human Machine Interface is the interface through which the user interacts with the system. The current status of the system is communicated to the user by means of a GUI. 
The user can also turn ON or OFF various functions from the interface. The HMI was created by Siemens WINCC V5.5. Tags were used to enable communication between the WINCC and the SIMANTIC Manger.

\section{Design And Implementation}

The overhead Tank is to be filled by a Pump. The pump will automatically start when the water level of Over Head Tank reaches below Low Level and stop when the level reaches High Level. Dry run is checked by the Low Level sensor of the Under Ground Tank. In that case Pump will not run. Run time monitoring of the pump in Second and minute is recorded and Reset Switch is also provided. Provision of Manual Start/Stop switch is incorporated which will totally override the automatic system. Provisions are also made for various alarms, such as "Underground Tank Empty Alarm"and "Pump run time exceeded 10 Minutes" (If pump runs continuously for 10 Minutes).

The implementation is divided into four parts:-

3.1 Sensor Positioning

3.2 Circuit Design

3.3 Ladder Programing

3.4 HMI Creation

\subsection{Sensor Positioning}

Four inductive Proximity sensors were used to sense presence of water at required levels. The sensors are UG_LL - Low Level Sensor Underground tank (I 0.2), OH_LL - Low Level Sensor Overhead tank (I 0.0), OH_HL - High Level Sensor Overhead tank (I 0.1) are placed as shown below in Fig 3.

(Theses sensors give open contact when they are inside water and they give close contact when they are outside water.)

Fig. 3 The Schematic Design of the System

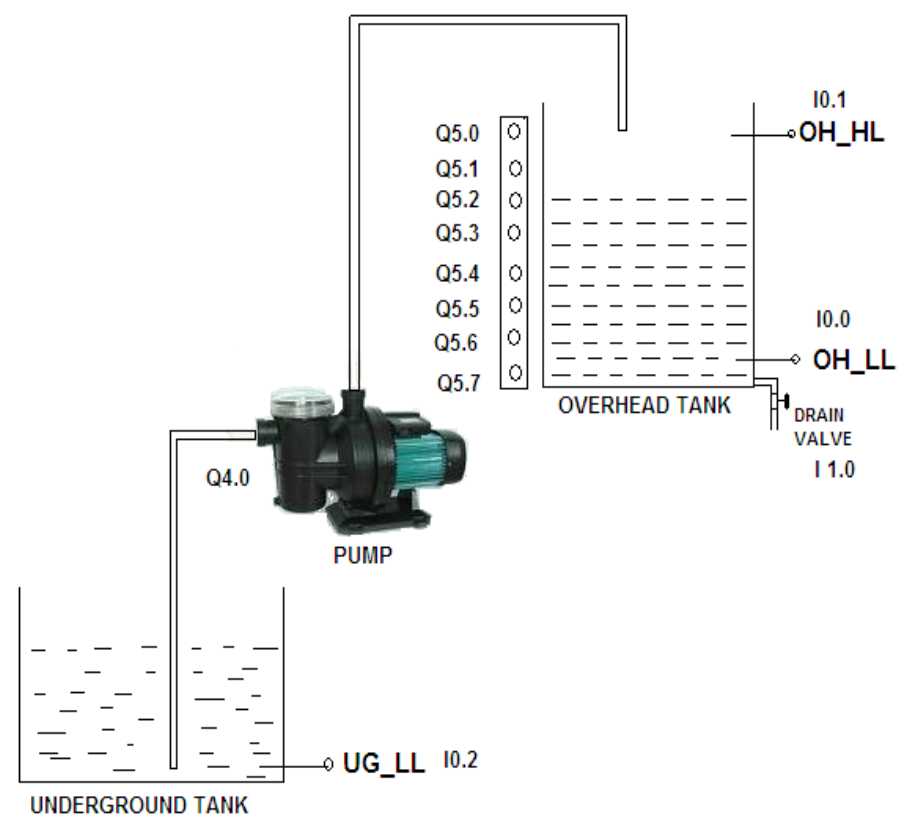

\subsection{Circuit Design}

DI or Digital Input provides a volt-free contact to the controller from the sensors. While DO or Digital Output provides the controller volt-free relay contact to control various equipment. One 16 bit DI and DO was sufficient for the successful completion of the project. Dl 16x DC 24V (order no.-6ES7-321-1BH01-0AA0) was used along with DO 16x DC 24V/0.5A (order no.-6ES7-322-1BH01-0AA0).PS 307 (order no.- 6ES7-3071BA00-0AA0) was used to power the PLC. The wiring diagrams of DI and DO are shown in Fig 4 and Fig 5 respectively. 


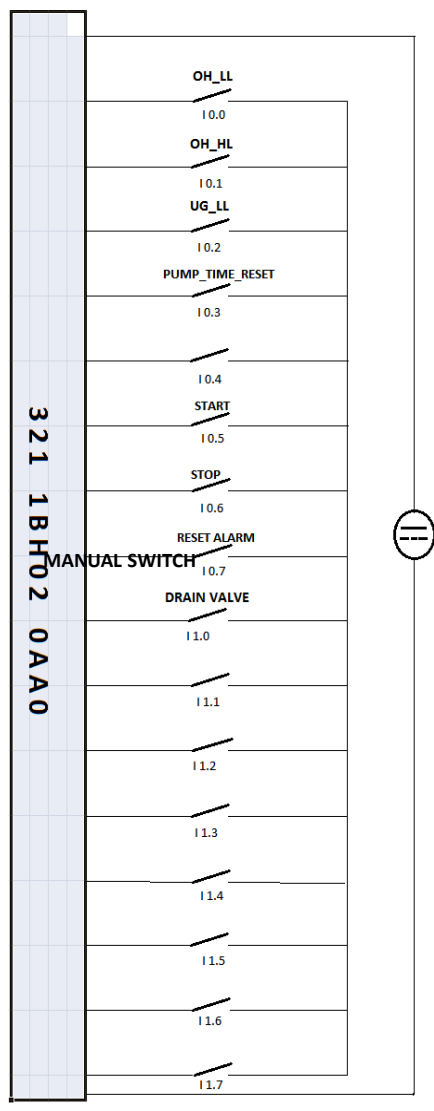

Fig.4 Wiring Diagram of 16 bit DI

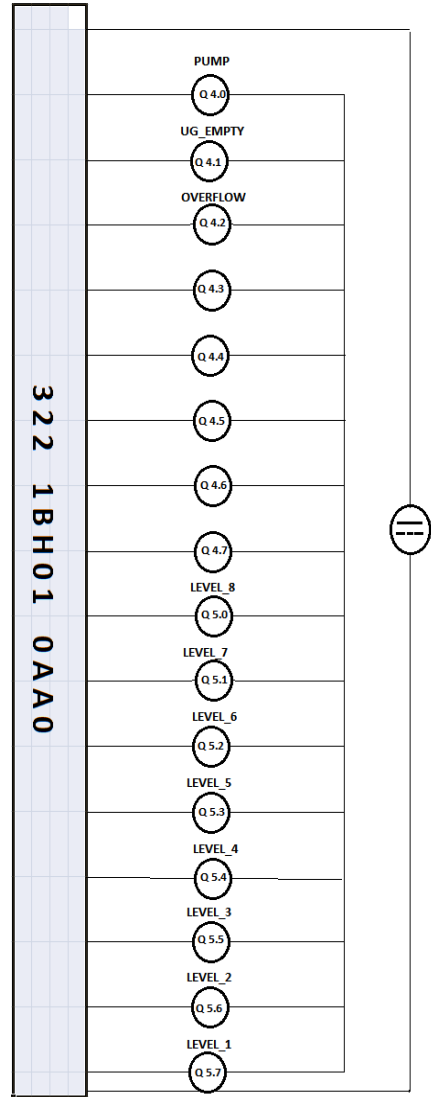

Fig. 5Wiring Diagram of 16bit DO

\subsection{Ladder Programming}

The Ladder programming for the Siemens S7-300 was done in Siemens Simantic manager [8]. The symbol used in the ladder logic is shown in the symbol table in Fig 7.
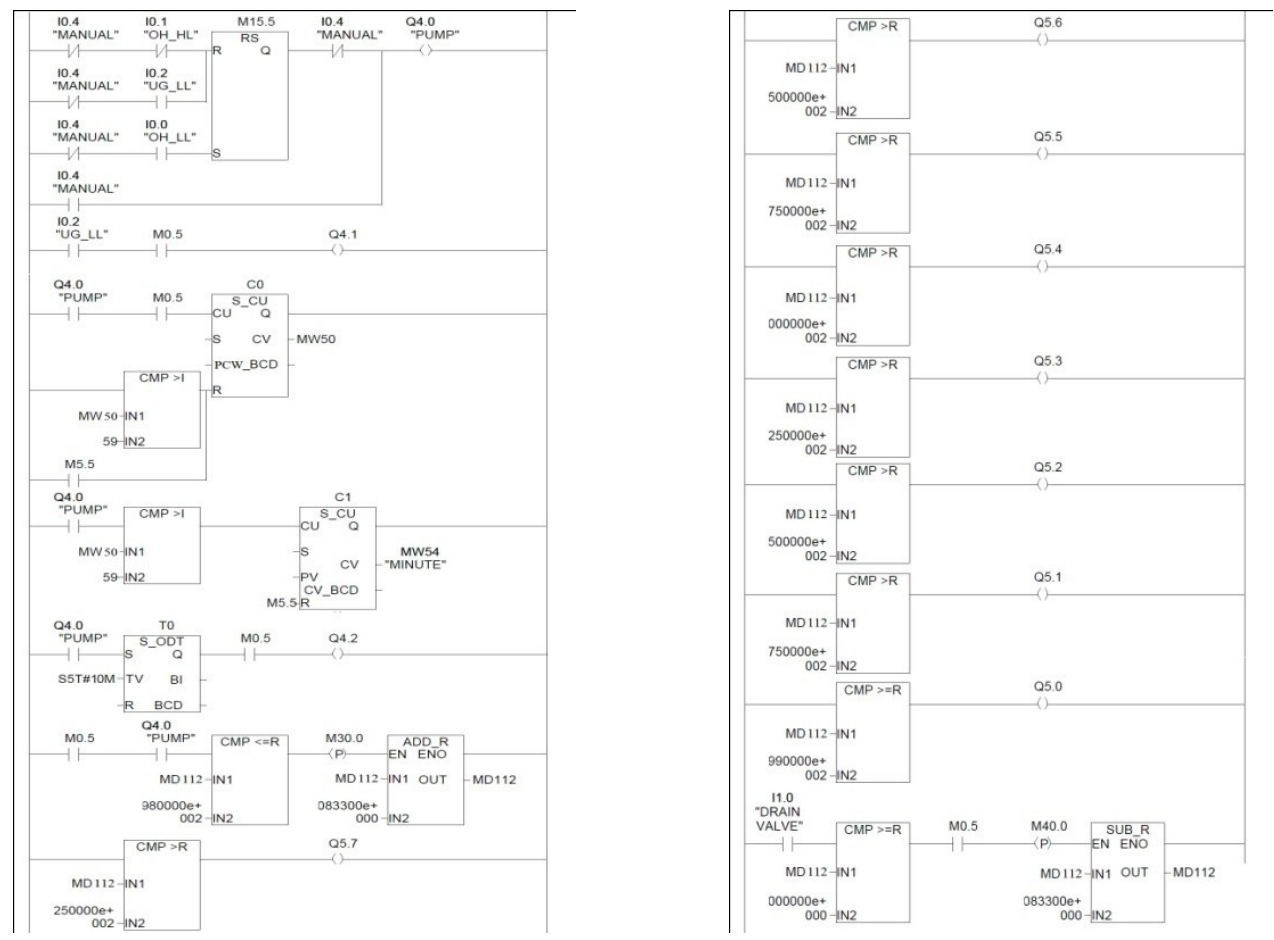

Fig. 6 The ladder logic 
The ladder logic shown in Fig 6 shows tank level control simulated for 1000 litre tank with a constant flow rate of 125 litres per min. The flow rate of the drainage is also taken as 125 litres per minute. The Symbol Table for the written ladder logic is shown above in Fig 6

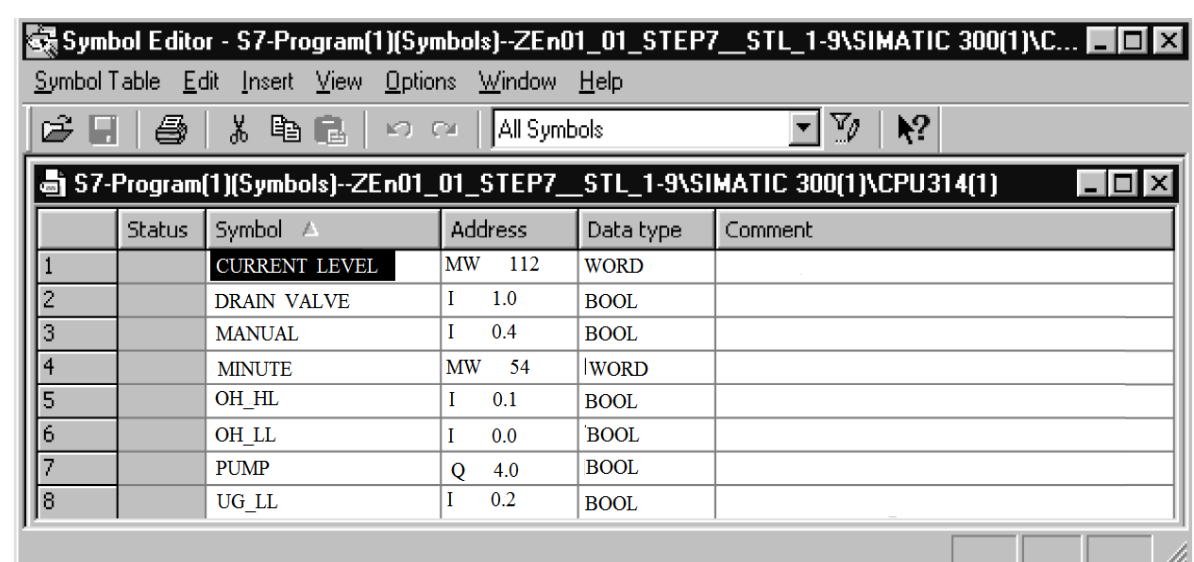

Fig. 7 Symbol Table for the ladder logic

\subsection{HMI Designing}

The Human Machine Interface was created in WinCCExplorer[9]-[10]. Tags were used for the communication of the PLC Ladder logic operation in the Simantic manger with the HMI. The created HMI is shown in Fig 8 below. The whole project is shown along with the sensor positions.

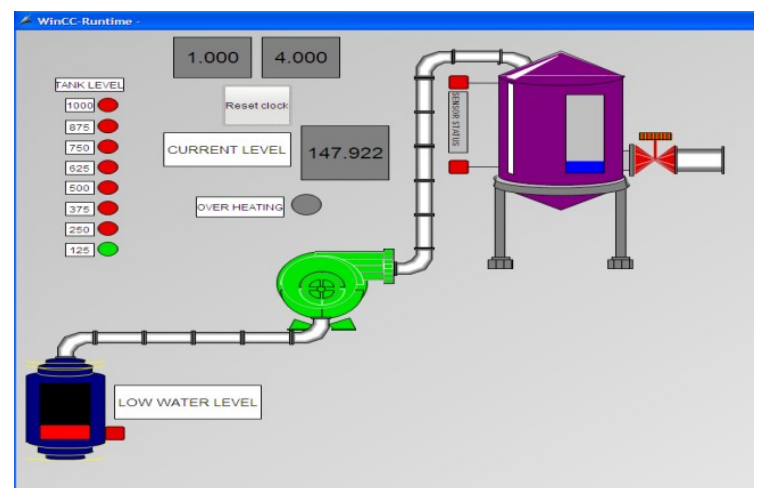

Fig. 8SCADA Interface for the Tank Level Control

\section{Results}

The results were taken in four situations, and were represented in Variable Table (VAT). The situations are

4.1 Empty tank

4.2 Filling tank

4.3 Full tank

4.4 Draining.

The variable description along with its status is shown. The manual override is turned OFF to enable automatic operation. 


\subsection{Empty Tank}

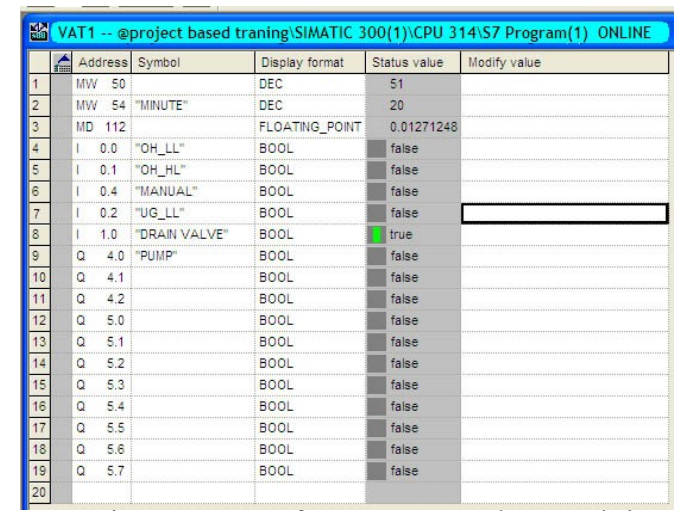

Fig. 9 Status of parameters when tank is empty

The Figure 9 shows the status of the various parameters when the tank is empty. The status of the level indicators from Q5.0 to Q5.7 is all FALSE denoting absence of water. The drain valve is true showing that the tank is being emptied. As drain valve is ON pump is switched OFF.

\subsection{Filling Tank}

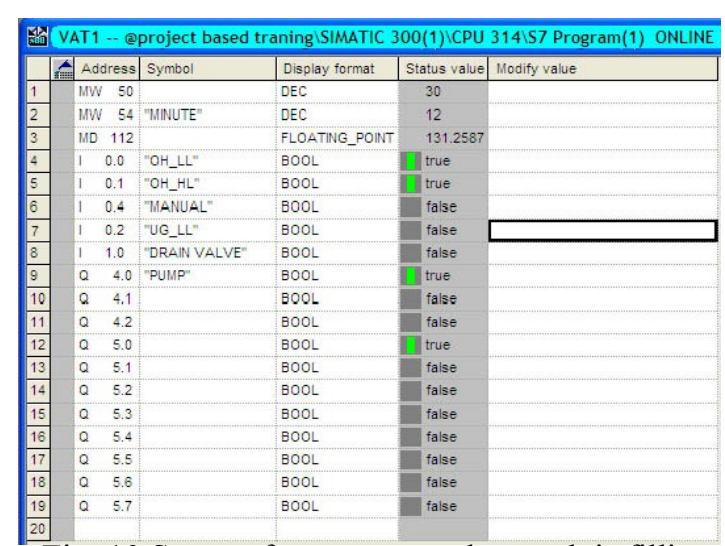

Fig. 10 Status of parameters when tank is filling

The Figure 10 shows the status of the various parameters when the tank is being filled. Pump is ON and Drain valve is OFF. The level of the increasing liquid is denoted by the indicators from Q5.0 to Q5.7.

\subsection{Full Tank}

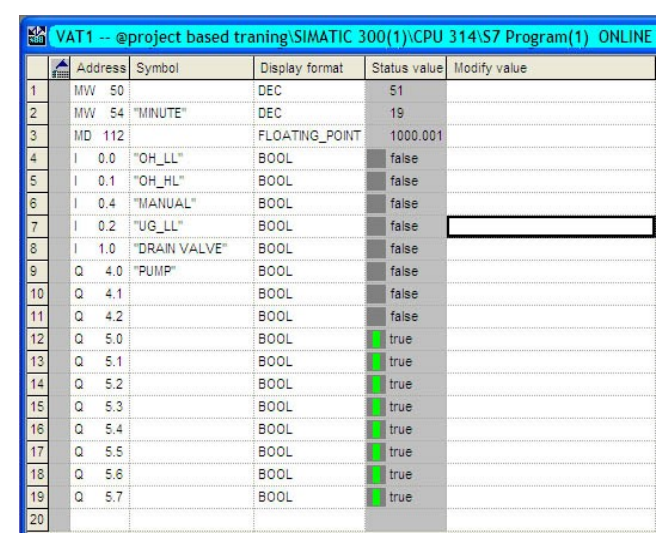

Fig. 11 Status of parameters when tank is Full

The Figure 11 Shows TRUE value for all the level indicators denoting that liquid is present in every level and tank is full. The pump is automatically turned OFF. The manual switch is kept OFF. 


\subsection{Draining}

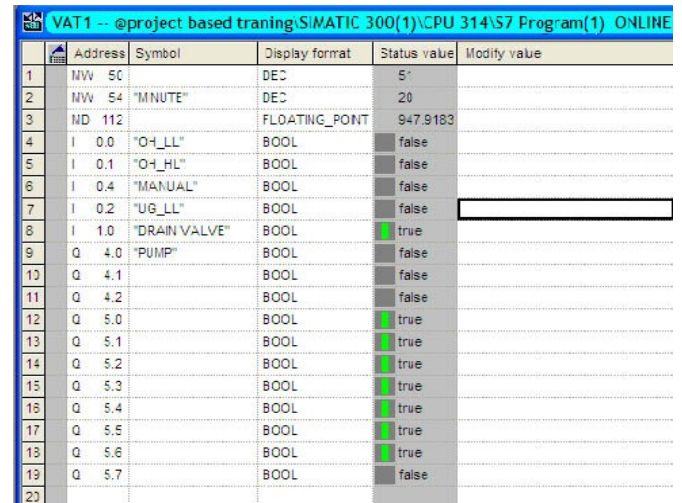

Fig. 12 Status of parameters when tank is Draining

During draining the drain valve shows TRUE value. The pump and the manual switch is kept OFF. The level indicators (Q5.0-Q5.7) show decreasing level.

UG_LL - Low Level Sensor Underground tank (I 0.2), OH_LL - Low Level Sensor Overhead tank (I 0.0), $\mathrm{OH} \_\mathrm{HL}$ - High Level Sensor Overhead tank (I 0.1) status is denoted in each of the figures. They give FALSE status when inside water.

\section{Conclusion}

Our project readily achieved its aim of automating the level control process. No human supervision was necessary. The system did not use float sensors yet denoted the accurate level. Hence the short coming of the float sensors that is unwanted vibrations and high cost was easily overcome. The PLC Siemens SIMANTIC S7-300 also offers many Input Output ports. Hence this single system can singlehandedly control as many as 50 tanks making it efficient and cost effective.

\section{Books:}

\section{References}

[1]. FestoDidactic ,Programmable Logic Controllers Basic Level(Textbook TP 301, GmbH \& Co., D-73770 Denkendorf.)

[2]. Bolton, Programmable Logic Controllers.(4th ed.W. (2006). North Carolina: Elsevier Newnes.)

[7]. Siemens AG,Working with step 7 V5.2, Getting Started(edition- December 2012)

[8]. Siemens AG,Ladder Logic (LAD) for S7-300 and S7-400 Programming, Reference Manual, (edition-December 2012)

[9]. Axel Daniels \& Wayne Salter, What is SCADA, (CERN-CNL-2000-003 Vol. XXXV, issue no 3.)

\section{Journal Papers:}

[3]. J.C.Chan, H.L.T.Dy, A.H.Fernando, R.L.Tiu, P.J.G.Viernes, "Design, Fabrication, and Testing of a PLC Training Module Using Siemens S7-300 PLC", DLSU Engineering e-Journal,Vol. 1 No. 1, March 2007, pp.43-54.

\section{Proceedings Papers:}

[4]. 2002 S.Ghosh, S.Bairagya, C.Roy, S.Dey, S.Goswami, A.Ghosh, ET \&TE-2008, "Bottle Filling System using PLC"

[5]. A.Saha, S.Kundu, A.Ghosh, MDCCT-2012, "MINI CEMENT PLANT USING PLC”, Conference proceedings, 6-7 february,2012, Burdwan University

[6]. Hao, L. and Ruilin, P. (2005). Application of Centralized PLC Automation Control in Painting Line of Steel Plant. Proceeding of the 4th Asian Conference on Industrial Automation and Robotics, Landmark Hotel, Thailand

\section{Theses:}

[10]. Tony Stafford, Taking Back - A SCADA case study, Camrosa Water District, California, July 2002 